\title{
Efecto de la poda sobre la cantidad y el rendimiento del aceite esencial de Laurus nobilis
}

\section{Pruning effect on essential oil Laurus nobilis quantity and performance}

Fecha de Recepción: 17 de Junio de 2013

Fecha de Aceptación: 16 de octubre de 2013

\author{
Ana María Callejas¹, Néstor Fabián Sierra², \\ Pedro José Almanza-Merchán ${ }^{3}$, Óscar Julio Medina ${ }^{4}$
}

\section{Resumen}

Objetivo. Conocer el comportamiento de la planta de laurel con diferentes podas, en cuanto a su rendimiento y cantidad de aceite esencial para satisfacer las demandas del mercado. Materiales y métodos. Se monitorearon 48 plantas, ubicadas en el municipio de Nuevo Colón, Boyacá, en un diseño completamenteal azar, con tres tratamientos (deshoje, despunte y el testigo sin poda) en 5 estadios fenológicos, con cuatro repeticiones. Se evaluó la respuesta en cuanto a tres solventes usados en la extracción y diferentes tipos de poda. Resultados. El hexano fue el solvente que permitió mayor extracción de aceite esencial de las hojas de laurel, seguido de etanol y cloroformo-metanol, respectivamente. Asimismo, en los diferentes tratamientos de poda se demostró que la mayor cantidad de aceite esencial $(p \leq 0,01)$ se obtuvo

\begin{abstract}
Objective. To find the laurel plant behavior, after different ways of pruning, in terms of performance and essential oil amount, to meet market demands. Materials and methods. 48 plants were monitored, located in the municipality of Nuevo Colón, Boyacá, on a completely randomized design, and with three ways of treatments (defoliation, bud break offs and control without pruning) in 5 phenological stages, and four replications. The evaluation response was related to the three solvents used in the extraction and different pruning types. Results. The hexane was the solvent which allowed the most amount of essential oil extraction from the leaves, followed by the ethanol and the chloroform-methanol, respectively. Also, it was demonstrated in the different trimming treatments, that the higher amount of essential oil
\end{abstract}

1 Ingeniera Agrónoma. Universidad Pedagógica y Tecnológica de Colombia, Tunja. anamariacallejas.leguiuzamon@hotmail.es

2 Ingeniero Agrónomo. Universidad Pedagógica y Tecnológica de Colombia, Tunja.nsfabian7@gmail.com

3 Docente, Investigador Grupo Ecofisiología Vegetal. Universidad Pedagógica y Tecnológica de Colombia, Tunja. ppcalma@gmail.com

4 Docente, Investigador, Grupo GIQTA. Universidad Pedagógica y Tecnológica de Colombia, Tunja. oscar.medina@uptc.edu.co 
en hojas de laurel con despunte, mientras que con deshoje se obtuvo la menor cantidad. Analizando el rendimiento, se encontraron rendimientos $(2,32 \%)$ en las hojas de laurel con despunte, seguido de las plantas testigo $(2,05 \%)$ y por último las plantas con deshoje $(1,24 \%)$. Conclusiones. El despunte, como práctica agronómica, en laurel representa mayores beneficios al productor y es una alternativa de valor agregado.

Palabras clave: Laurel, solventes, aceite esencial, despunte, deshoje (Fuente: USDA). extraction $(P \leq 0.01)$ was obtained with tipping bay leaves, while with defoliation was obtained smaller amounts. Analyzing the performances, we found $(2.32 \%)$ yields in the bay leaves with topping followed by the plants control $(2.05 \%)$ and finally by plants defoliations $(1.24 \%)$. Conclusions. It was found that the pruning practice in the laurel plantations, represents greater benefits to the producer and is a value-added alternative.

Key words: Laurel, solvents, essential oil extraction, topping, defoliation (Source: USDA). 


\section{Introducción}

El laurel (Laurus nobilis) es un árbol de hoja perenne que pertenece a la familia Lauraceae y comprende 32 géneros y de 2000 a 2500 especies; es originario del sur del mediterráneo y cultivado en muchas regiones templadas y cálidas del mundo, particularmente en la zona del Mediterráneo (Turquía, Grecia, España, Portugal, Marruecos y México) y en EE.UU. como planta ornamental (1). En Turquía se cultiva de forma nativa desde la costa hasta una altitud de 600-800 msnm (2).

Los aceites esenciales de L. nobilis son de gran valor y se utilizan como materia prima de perfumes, cosméticos, especias y productos de aromaterapia, fitoterapia y nutrición (3). Las hojas se han utilizado para tratar diversas enfermedades, como la epilepsia, el parkinson (4) y neuralgias; además, se ha mencionado que tiene propiedades antibacterianas, antifúngicas, antivirales, insecticidas y antioxidantes (5).

Existen numerosos estudios sobre la composición química de los aceites esenciales obtenidos a partir de las hojas de $L$. nobilis de las regiones mediterránea y europea $(6,7)$, en los cuales se mencionan que los principales componentes del aceite esencial son cineol $(44,12 \%)$, eugenol $(15,16 \%)$, sabineno $(6,20 \%)$, 4-terpineol $(3,60 \%)$, a-pineno $(2,74 \%)$, metileugenol $(2,48 \%)$, a-terpineol $(2,19 \%)$ y A-pineno $(2,05 \%)$. Sin embargo, diferentes estudios han reportado importantes diferencias en la composición química del aceite según la variedad, los métodos de cultivo, la época de cosecha y la procedencia geográfica de la planta (8).

En Colombia, el laurel (Laurus nobilis L.) se cultiva con fines de exportación en diferentes regiones del país y mediante diferentes métodos agronómicos, evidenciándose cambios en los tiempos de rebrote y en el tamaño de las hojas, lo que tendría consecuencias sobre la productividad del aceite; sin embargo, son muy pocos los estudios que reporten el efecto de actividades como la poda que regula la capacidad vegetativa y reproductiva de la planta- sobre el rendimiento del aceite esencial.
Con el objetivo de brindar una herramienta más a los productores de laurel y determinar cómo influye la poda sobre el contenido de aceite esencial, se desarrolló un estudio en diferentes plantas de laurel bajo las condiciones edafoclimáticas del municipio de Nuevo Colón, Boyacá.

\section{Materiales y métodos}

Localización y características geoclimáticas. El trabajo se desarrolló en la finca El Loro, vereda de Llano Grande, en el municipio de Nuevo Colon, ubicado en el departamento de Boyacá; su cabecera está localizada a los $05^{\circ} 21^{\prime} 30^{\prime \prime}$ de latitud norte y $73^{\circ} 27^{\prime} 38^{\prime \prime}$ longitud oeste; se presenta una temperatura promedio de $12{ }^{\circ} \mathrm{C}$, una temperatura mínima promedio de $14.4{ }^{\circ} \mathrm{C}$ y una máxima de $6.5^{\circ} \mathrm{C}$, valores anuales, y su precipitación tiene un comportamiento de tipo monodal, con promedios de $912.4 \mathrm{~mm}$ anual (IDEAM 1980-1998). El municipio de Nuevo Colón se encuentra a una altura de $2500 \mathrm{msnm}$; debido a que su territorio se encuentra en la Cordillera Oriental, en las estribaciones del altiplano Cundiboyacense, sobre colinas denudacionales conformadas por materiales y geoformas que originan terrenos quebrados y modelados por las condiciones locales de clima frío, se encuentran suelos de orden Andisol, con buenas propiedades fisicoquímicas (9).

Material vegetal. Las plantas seleccionadas se encontraban sembradas a 1,5 m entre surcos, y $1 \mathrm{~m}$ entre plantas, las cuales fueron marcadas con ficha técnica y un lazo de diferente color, dependiendo del tratamiento: rojo para despunte, verde para deshoje y amarillo para testigo. Se procedió a medir variables como la altura y el número de brotes, iniciales (0 días), y a aplicar los diferentes tratamientos de poda.

Trabajo de campo. Se instaló un Datologger climatologic en la finca, con el cual se hizo la lectura diaria de temperatura y humedad relativa a las 7 a.m., 1 p.m. y 7 p.m., para comparar el efecto de la temperatura con el desarrollo de los brotes y hojas; de esta manera, a los 60, 90, 120 y 150 días de la aplicación de los tratamientos se tomaron datos fenológicos, siendo constante la observación 
de los cambios en las plantas. La recolección del material vegetal se hizo bajo las condiciones de los subestadios fenológicos determinados en el trabajo de investigación; este material fue recolectado en campo a los 60, 90, 120 y 150 días después de poda de las plantas de laurel.

Métodos de laboratorio. El material recolectado fue Ilevado al laboratorio del Grupo de Investigación Química y Tecnología de Alimentos, GIQTA, de la Universidad Pedagógica y Tecnológica de Colombia, a fin de efectuar el análisis fisicoquímico, que estuvo dividido en dos partes. La primera parte fue la extracción del aceite esencial de las hojas de laurel en el Laboratorio, empleando el método de extracción con solventes guiado por microondas (Sineo, MDS-86), y la medición del rendimiento (peso del extracto obtenido sobre el peso de material vegetal utilizado por 100). Para el proceso inicial se tomaron $500 \mathrm{~g}$ de hojas de laurel testigo, limpio y clasificado, descartando presencia de pústulas, hongos, impurezas y mordeduras de insectos; estas hojas se llevaron a mufla durante 36 horas a $60{ }^{\circ} \mathrm{C}$, y en estado seco se pulverizaron. A continuación se pesaron y se realizaron sendos ensayos con etanol, cloroformo-metanol y hexano, adicionándole a cada uno $2.5 \mathrm{~g}$ de laurel, con tres réplicas de cada uno; el siguiente paso fue ingresarlos al microondas (las condiciones de la extracción fueron $70{ }^{\circ} \mathrm{C}$, 10 segundos y $700 \mathrm{~W}$ ); luego, se procedió a filtrar lo residuos de hojas, y las muestras se dejaron en refrigeración durante 12 horas; se filtró nuevamente $y$, finalmente, se dejó secar durante 12 horas a $30{ }^{\circ} \mathrm{C}$, para obtener el aceite esencial, y realizar los cálculos de rendimientos de los diferentes solventes.

En la segunda parte se recolectaron hojas de las plantas testigo, que fueron secadas a temperatura ambiente durante ochos días y enviadas al Laboratorio de Cromatografía y Espectrometría de Masas, de la Universidad Industrial de Santander, donde re realizó la extracción de aceite mediante la metodología de hidrodestilación, y posterior determinación (identificación tentativa y cantidad relativa, \%) de la composición química de muestras de aceite esencial de laurel, utilizando cromatografía de gases acoplada a espectrometría de masas (GC-MS)*1 (impacto de electrones, 70 $\mathrm{eV}$ ) operado en el modo de barrido completo de radiofrecuencia (full scan).

Diseño experimental. Se estableció un diseño completamente al azar (DCA), con 2 tratamientos (despunte y deshoje) y un testigo (plantas sin poda), evaluándose 5 épocas, que correspondieron a diferentes estadios fenológicos (60, 90, 120 y 150 días después de poda) y un tratamientos inicial válido para todas las variables; cada tratamiento se replicó cuatro veces. Cada unidad experimental estuvo conformada por plantas de laurel de 4 años de edad, para un total de 48 unidades experimentales.

Análisis estadístico. Todos los datos obtenidos en las diferentes pruebas a lo largo del estudio fueron consignados en libretas de campo, analizados mediante el programa estadístico SAS $\vee 9.2$ y representados en diferentes tablas y figuras, tras un análisis de varianza para el diseño al azar. Para determinar si había diferencias entre promedios se realizó la prueba de comparación de medias de Tukey $(p>0,05)$.

\section{Resultados y discusión}

Eficiencia de los métodos de extracción. Gran variedad de solventes orgánicos suelen ser utilizados para la extracción de aceites, siendo los más populares el hexano y el etanol, mediante una mezcla, con la cual es posible extraer hasta el $98 \%$ de los lípidos presentes en la biomasa (10). Con base en lo anterior, se encontró que con diferencias significativas ( $p \leq 0,01)$, el hexano fue el solvente que permitió mayor extracción de aceite esencial de las plantas de laurel, seguido de etanol y cloroformo-metanol, respectivamente. 


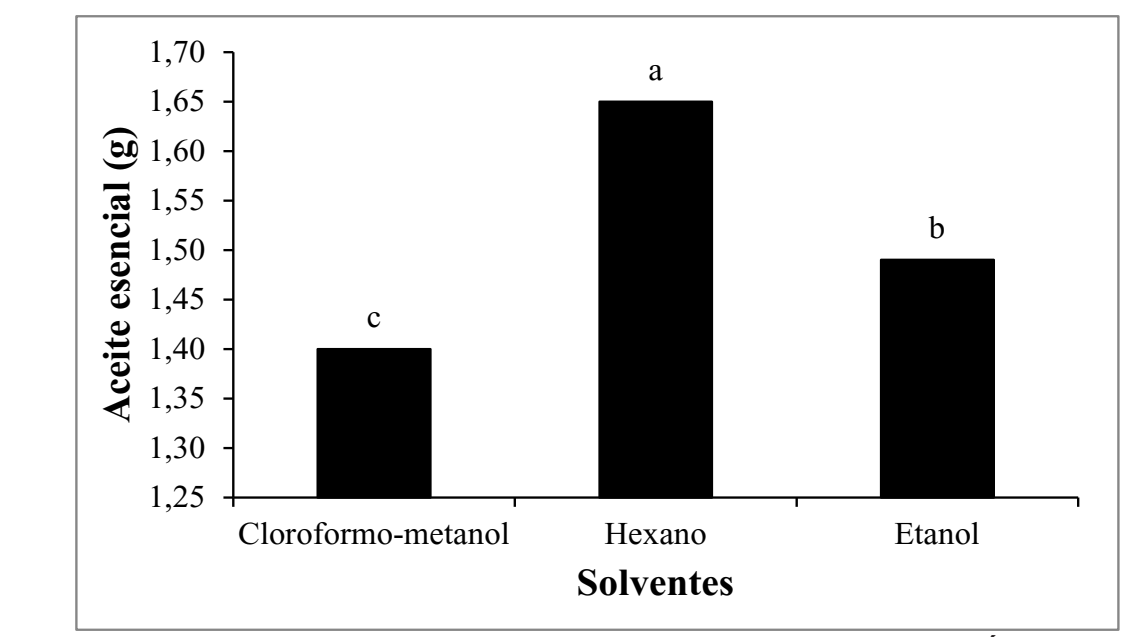

\section{FIGURA 1. EFECTO DE LOS SOLVENTES SOBRE LA EXTRACCIÓN DE ACEITE ESENCIAL EN PLANTAS DE LAUREL. Letras diferentes indican diferencias significativas $(p \leq 0,01)$}

Esto concuerda con lo reportado por Can (2010), quien sostiene que el hexano es un solvente ideal para realizar trabajos delicados en los que la identidad de los componentes puede resultar comprometida. Así mismo, la producción se da con las condiciones de pureza adecuadas, y los residuos en la extracción son pocos por el punto de ebullición que maneja, el cual es altamente alejado del punto de ebullición de los aceites esenciales. Contreras y Ruiz (2012) encontraron que el solvente en mención permite determinar las propiedades físicas del aceite esencial presente en la cáscara de pomelo (Citrus máxima), logrando un rendimiento apreciable respecto a otro método de extracción, siendo el hexano el solvente más eficiente.

Es de mencionar, además, que según García y Miranda (2011), quienes compararon diferentes métodos de extracción en microalgas para la producción de biodiésel, el hexano generó mejores porcentajes de extracción. De igual forma, se determinó que la extracción de aceite de microalga utilizando como solvente el hexano produce menores impactos ambientales en comparación con las mezclas con solventes etanol-hexano y metanol-cloroformo, debido a la alta toxicidad que representan estas mezclas. Esto confirma lo señalado por Manahan (2007), quien menciona que el etanol es un compuesto muy volátil, por lo cual, al pasar el aire, se fotooxida a acetaldehído, cuya oxidación conduce a la formación de nitrato de peroxiacetilo (PAN), que tiene toxicidad más alta para las plantas y puede ocasionar daño en la vegetación. Esto hace que la elección del solvente de extracción no solo se base en su capacidad extractiva, sino en el impacto que pueda tener en el medioambiente; de allí que el solvente más eficiente es el hexano.

Cantidad y rendimiento del aceite extraído en relación con diferentes tipos de poda. La mayor cantidad de aceite esencial $(0,059 \mathrm{~g})(\mathrm{p} \leq 0,01)$ se obtuvo en plantas de laurel con despunte, mientras que con deshoje se obtuvo la menor cantidad $(0,032 \mathrm{~g})$, encontrándose diferencias estadísticamente significativas (Figura 2). De otra parte, se determinó que el mayor rendimiento en la extracción de aceite esencial $(p \leq 0,01)$ se obtuvo en plantas de laurel con despunte $(2,32 \%)$, seguido de las plantas testigo y por último las plantas con deshoje $(1,24 \%)$ (Figura 3). 


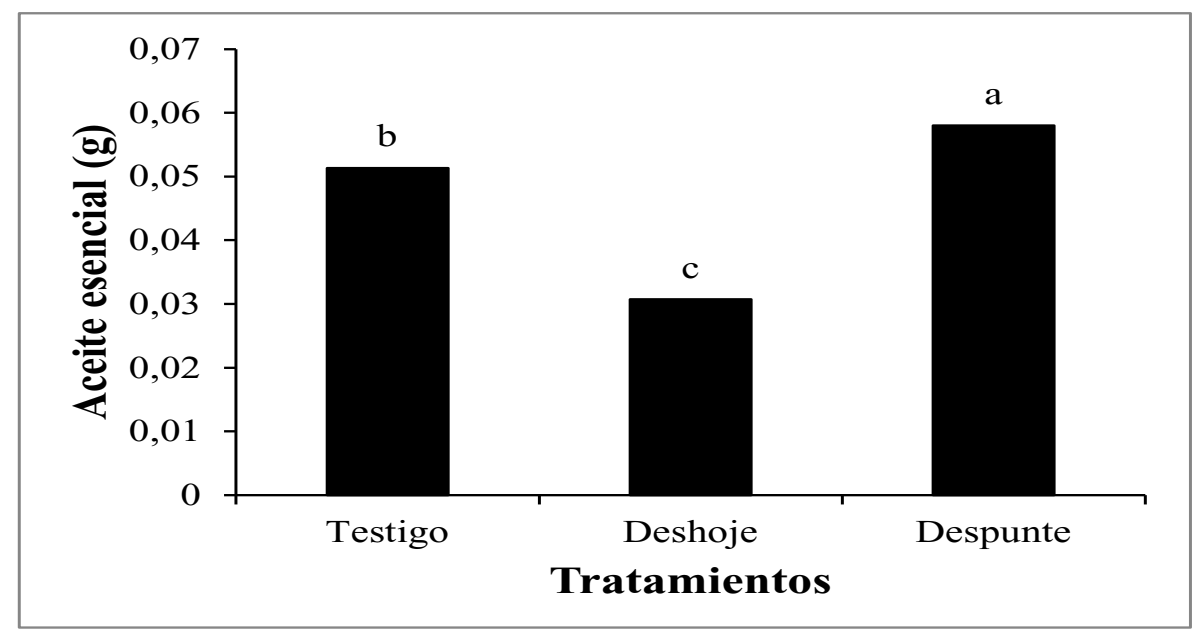

FIGURA 2. CANTIDAD DE ACEITE ESENCIAL EN HOJAS DE LAUREL. Letras diferentes indican diferencias significativas $(p \leq 0,01)$

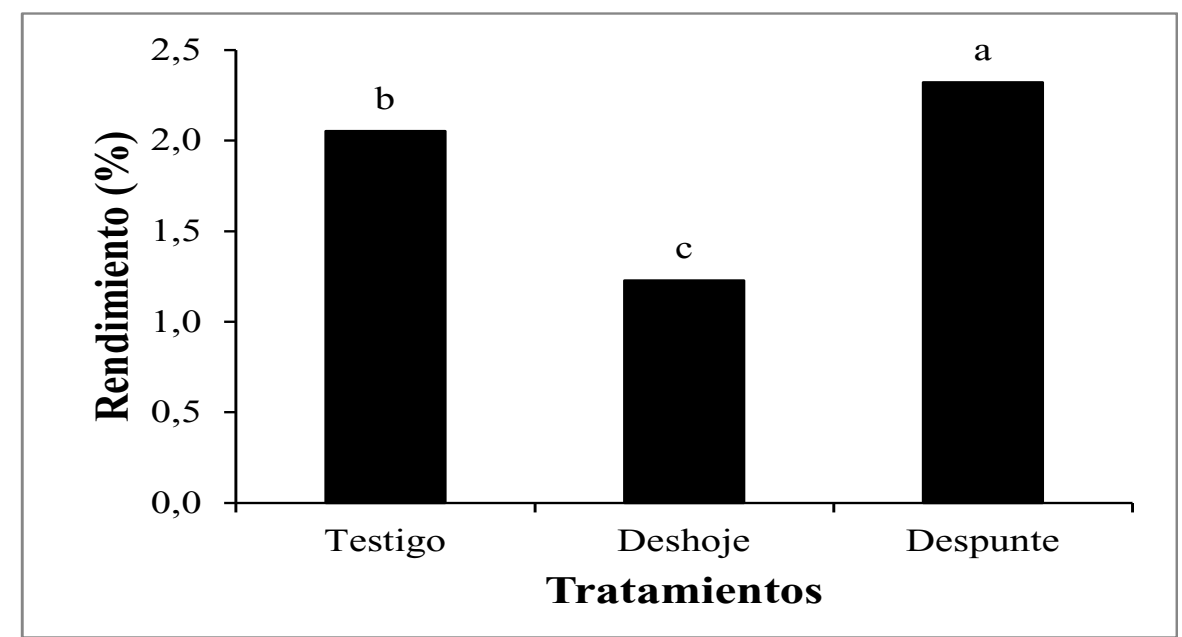

FIGURA 3. EFECTO DEL DESPUNTE Y EL DESHOJE SOBRE EL COMPORTAMIENTO DE LA MASA FRESCA DE PLANTAS DE LAUREL. Letras diferentes indican diferencias significativas $(p \leq 0,01)$

Los factores más importantes que influyen sobre la composición y el rendimiento de los aceites esenciales son el método de cultivo, los fertilizantes y abonos utilizados, las distancias de siembra y las podas; asimismo, las condiciones geobotánicas -clima, altitud, tipo de suelo, luminosidad, pluviosidad y temperatura-, la época de recolección, la edad la planta y los métodos de extracción del aceite tienen algún efecto sobre estos aspectos (15).

Igualmente, es importante indicar que el rendimiento de aceite esencial se ve afectado porque durante el cortado (poda) se rompen células que contienen el aceite esencial y porque durante el secado se merma debido a su alta volatilidad 
(16). Teniendo en cuenta lo anterior, se puede afirmar que las diferencias en cuanto a la cantidad y el rendimiento del aceite de laurel respecto a los dientes tipos de poda se pueden atribuir a diversos factores. Aún es muy escasa la literatura que reporte los cambios fisiológicos que sufren las plantas tras la poda en las condiciones específicas de Nuevo Colon; sin embargo, es importante mencionar que la poda y, particularmente, el deshoje provocan daños estructurales en las plantas que afectan el rebrote, lo que puede traer consecuencias en la cantidad y calidad del aceite producido. De igual forma, el despunte permite un mejor índice de crecimiento de la planta y de área foliar (17), lo que podría tener alguna relación con la cantidad de aceite extraído de las hojas de laurel, dado que la planta logra un mejor nivel de recuperación y mantiene hojas maduras con buena cantidad de aceite.

En otros estudios en gramíneas, Wallace y col. (1984), citados por Reyes y Martínez (2001), al aplicar tres niveles de corte $(25 \mathrm{~cm}, 36 \mathrm{~cm}$ y 54 $\mathrm{cm})$ registraron diferencias en la conductancia estomática, la transpiración y la fotosíntesis, afectando la morfología y el área foliar, lo cual podría estar ligado a la disminución de la capacidad biosintética de algunos compuestos, como es el caso de los aceites.

De otra parte, Bruce (2006) menciona que prácticas como el deshoje total provocan mayor agotamiento de la reserva de nutrientes, así como la incapacidad de la planta para la fabricación de energía, lo que debilita el árbol y trae como consecuencia el retraso en el crecimiento, presencia de hojas débiles y de color verde pálido $y$, posiblemente, muerte progresiva de la rama. Adicionalmente, señala que los arbustos también cambian fisiológicamente tras la defoliación, pues la producción de sustancias protectoras que ayudan a la resistencia a enfermedades puede ser inhibida, lo cual se confirma con lo encontrado en este estudio, en donde el deshoje tendría entonces un mayor impacto sobre la capacidad de recuperación de la planta de laurel y, por ende, sobre la cantidad y el rendimiento del aceite producido.

Así, con los resultados obtenidos en este estudio se evidencia que existe diferencia significativa entre los métodos de extracción utilizados, siendo este un resultado favorable para la toma de decisiones en la transferencia de tecnología del proceso de produccion del aceite esencial de laurel, debido a que, teniendo en cuenta los factores ambientales, energéticos y económicos, es mejor utilizar el hexano como solvente, comparado con los otros dos, los cuales se descartan por su baja eficiencia en la extracción.

En cuanto a los tipos de poda, se estableció que esta variable ejerce influencia sobre la cantidad y el rendimiento del aceite esencial extraído de las hojas de laurel; sin embargo, es poca la literatura que reporta el efecto directo que tendría la poda sobre estos dos aspectos. Este trabajo abre nuevas líneas de investigación en cuanto a los efectos que tienen específicamente el manejo agronómico y las características geográficas de Nuevo Colon sobre la calidad y cantidad del aceite esencial de laurel.

\section{Referencias bibliográficas}

(1) Barla A, Topcu G, Oksuz S, Tumen G, Kingston D. Identification of cytotoxic sesquiterpenes from Laurus nobilis L. Food Chem 2007; 104: 1478-1484.

(2) Davies N. Gas chromatographic retention indices of monoterpenes and sesquiterpenes on methylsilicone and carbowax $20 \mathrm{M}$. phases. J Chromatog 1990; 503: 1-24.

(3) Buchbauer G. The detailed analysis of essential oils leads to the understanding of their properties. Perf Flav 2000; 25: 64-67.

(4) Aqili-Khorasanin MS. Collection of drugs (Materia media). Enqelab-e-Eslami Publishing and Educational Organization 1992: 624-630.

(5) Burt S. Essential oils: their antibacterial properties and potential applications in foods: a review. Inter J. Food Microbial 2000; 94: 223-253.

(6) Riaz M, Ashraf C, Chaudhary F. Studies of the essential oil of the Pakistani Laurus nobilis Linn in different seasons. Pak. J. Sci. Indust 1989; 32: 33- 35. 
(7) Mohammadreza V. Phenological Variation of Laurus nobilis Essentials Oil from IRAN. Elect J Environmen Agr Food Chem 2008; 7(11): 3321-3325.

(8) Rohloff J, Dragland S, Mordal R, Iversen T. Effect of harvest time and drying method on biomass production, essential oil, and quality of peppermint (Mentha x piperita L.). J Agr Food Chem 2005; 53(10): 4143-4148.

(9) Alcaldía de Nuevo Colón. Plan de Ordenamiento Territorial (POT). Nuevo Colón, Boyacá; 2000.

(10) Richmond, A. Handbook of microalgal culture: Biotechnology and applied phycology. WileyBlackwell Publishing, Estados Unidos; 2004.

(11) Can B, Buchbauer C. Handbook of Essential Oils 2010: 114-116.

(12) Contreras PE, Ruiz JD. Estudio comparativo de dos métodos de extracción para el aceite esencial presente en la cáscara de pomelo (Citrus maxima). Facultad de Ingeniería, Universidad de Cartagena; 2012.
(13) García H, Miranda JH. Comparación de métodos de extracción de aceite de microalgas a escala laboratorio para la producción de biodiesel, Facultad de Ingeniería, Universidad de Santander; 2012.

(14) Manahan SE. Introduccion a la química ambiental. Primera edición. Editorial Reverte, México; 2007.

(15) Stashenko E, Combariza J, Puertas M. Aceites esenciales: técnicas de extracción y análisis. Laboratorio de Cromatografia, Universidad Industrial de Santander; 1998.

(16) Vásquez O, Alva A, Marreros J. Extracción y caracterización del aceite esencial de jengibre (Zingiber officinale). Rev Amazon Inv Alim 2001; 1(1): 38-42.

(17) Reyes JM, Martínez MD. La plasticidad de las plantas. Elementos 2001; 41: 39-43.

(18) Bruce R. Defoliation and its effect on tree vitality. Bartlett Tree Research Laboratory Technical Report; 2006. 